\title{
The Effect of Noise and Finite Sampling on the Line Profile Variations of $m=0$ Modes
}

\section{H. Montgomery \\ Institut für Astronomie, Universität Wien, Türkenschanzstrasse 17, A-1180 Vienna, Austria}

Figure 1 is of the same form as Fig. 3 in Schrijvers et al. (1997): the upper part of each panel is the amplitude of the variation across the line at the mode frequency and the lower part is the phase shift of these two components across the line (in units of $\pi$ ). The parameters $\left\{V_{\mathrm{avg}}, k, \Omega / \omega^{0}, W\right\}$ were chosen to have values representative of the $\delta$ Scuti star $4 \mathrm{CVn}$. Each panel in this array of plots has $m=0$, with a value of $\ell$ of 0,1 , or 2 , and an inclination angle $\theta_{\mathbf{i}}$ between $10^{\circ}$ and $80^{\circ}$. In order to generate the synthetic line profiles, we use sampling times taken from the last week of the $1996 \delta$ Scuti campaign on $4 \mathrm{CVn}$, and we assume that the signal-to-noise ratio of the spectrum is 250 .

As the figure shows, regardless of the value of $\ell$ or $\theta_{\mathrm{i}}$, the amplitude and phase still display the common signature of $m=0$ modes: the amplitude at the mode frequency goes to zero at the line center while its phase simultaneously undergoes a $\pi$ phase shift, also at the line center. Thus, a multi-site spectroscopic campaign even as short as one week should be sufficient to detect the $m=0$ modes which have photometrically detected frequencies. These modes are important for theoretical modeling, since they are much less affected by uncertainties in the rotation rate than are other modes.

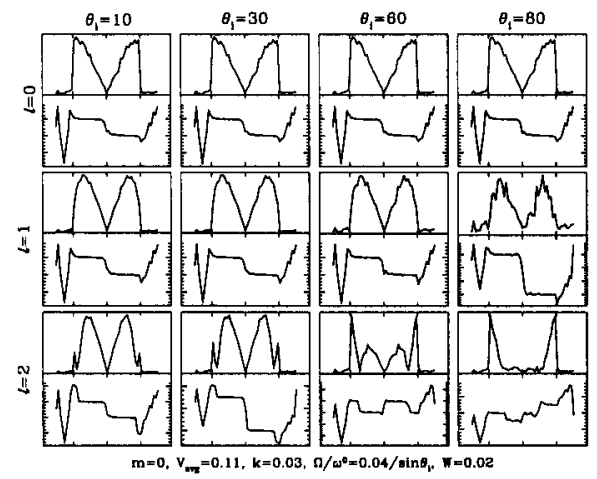

Figure 1. The amplitude and phase of LPVs of $m=0$ modes, for an array of $\ell$ and $\theta_{\mathrm{i}}$ values. Each panel shows the amplitude variation (upper curve) and phase variation (lower curve) across the line.

\section{Reference}

Schrijvers, C., Telting, J. H., Aerts, C., Ruymaekers, E., \& Henrichs, H. F. 1997, A\&AS, 121, 343 